\title{
Nanotechnology for cultural heritage
}

\author{
Santina Di Salvo \\ Department of Architecture (DARCH), University of Palermo, Italy \\ Email address: \\ santina.disalvo@unipa.it
}

To cite this article:

Santina Di Salvo. Nanotechnology for Cultural Heritage. International Journal of Science, Technology and Society.

Vol. 2, No. 2, 2014, pp. 28-32. doi: 10.11648/j.ijsts.20140202.12

\begin{abstract}
The conservation science is one of the most complex topics in the materials science as it requires interdisciplinary expertise ranging from the architecture, the technology of materials to the advanced analytical and physical chemistry. However, the recent development has shown that the complex tasks of the conservation of the cultural heritage can be solved very effectively using novel nanomaterials and nanotechnology methods.
\end{abstract}

Keywords: Patent, Consolidation, Protection, Nanostructured Materials

\section{Introduction}

Nanotechnology is considered as a new technological revolution, one so profound that will touch all the aspects of human society. Research groups believe that nanotechnologies could be successfully applied for the conservation of cultural heritage which is the base of our history and culture. In the past 20 years many researches have focused on developing suitable materials for consolidating and protecting materials as well as structures and many materials have been used with varying degrees of success, but the result of most of this methods is the increasing of decay or the rejection of the intervention.

In this study reports the Author was focused on the problems in conservation of ancient materials, identifying some possibilities in the applications of nanotechnologies in the field of conservation of architectural cultural heritage. Nanoparticles have dimensions that are less than $100 \mathrm{~nm}$. As a result of their very small size, they possess different properties to their corresponding bulk material. Their small dimensions are responsible for their large surface area, and this in turn results in a considerably increased surface to volume ratio, which then affects the nanoparticles physico-chemical properties. In particular they have distinctive optical, electronic, electrical, magnetic, chemical and mechanical properties.

This paper addresses to the latest innovative processes and methods, highlighting the issue on selecting most appropriate strategies and tools to achieve the recovery of identity and sustainability of interventions in the ancient built heritage, both at national and European level. The aim of this contribution is to evaluate the effectiveness of inorganic compatible treatments, based on nanosized particles of silicon dioxide as consolidants for ancient stones and wooden surfaces affected by different kinds of decay. Both in situ and laboratory tests were carried out and the re-aggregating effects of the deposited phase were investigated by superficial area analyses (BET) and SEM-EDX; it was also possible to obtain an estimation of the depth of penetration of the product inside the porous matrix. All the results converged in individuating these nanometric particles as an innovative, completely compatible, and efficient material for the consolidation of ancient and architectural surfaces.

\subsection{Background}

The last decade has witnessed an exponential growth of research activities based on studies on the preservation and appreciation of the ancient structures of cultural heritage, particularly of ancient stone and wood [1]. The theme of recovery of old buildings, applying non-traditional technologies, requires, in particular, a methodology to achieve goals that nowadays are consistent with the concept of sustainability. Recently, sustainability has evolved as a significant mode of thought in nearly every field of intellectual activity [2].

In this context, the protection and appreciation of the ancient materials like wood and stone, is of particular interest, especially through new technologies. Innovative technologies and, in particular, nanotechnology are multidisciplinary issues involving various research topics: from molecular biology to chemistry, from materials science 
to physics, and engineering [3]. Therefore, activities for the conservation of built heritage must be the result of synergies and collaboration of multiple groups, science and knowledge: architecture, engineering, technology, sociology, economics, urban planning, legislation must be managed and planned to work together. In fact, if sustainability ultimately means learning to think and act in terms of guaranteeing the prosperity of interdependent natural, social, and economic systems, then the built cultural heritage, with its unique values and experiences must be contextualized and integrated with this perspective [4].

We know that decisions concerning conservation of the built environment have in the past been the domain largely of architectural historians, urban planners, conservation specialists, and related professionals. Actually, conservation cannot remain a closed and solely self-referential profession, and indeed it has not [5]. With particular regard to the conservation of historic neighbourhoods and city centres, as well as individual monuments and sites of unique beauty, the challenge facing the conservation community is to develop a set of strategies and priorities that will permit it to focus its efforts on the conservation of those resources where the benefit-cost ratio is most favorable [6].

In this case sustainability means controlling change and choosing directions that capitalize most effectively on the inheritance from the past. In any decision about change and about the impact of the future on the remains of the past, therefore we should be conscious of two separate questions: the first is how to reconcile minimizing loss with the needs of the present; the second is how to ensure that the balance we strike does not reduce too greatly our successor's options for understanding and enjoying their inheritance.

Actually, built environment and heritage conservation should provide a dynamic vehicle by which individuals and communities can explore, reinforce, interpret and share their historical and traditional past and present, through community membership as well as through input as a professional or non-professional affiliate [7].

\section{State of the Art}

\subsection{Patents and Experimentations}

The use of innovative technologies can be a test and a challenge for the re-construction of the rules of the upgrade and the ancient built and buildable landscape, by inserting the environmental variable and the resulting constructive solutions [8]. The joint approach of different disciplines is a strength of the methodology of the project, where a large number of transversal phases can allow research groups to join in the development of activities that are useful to compare experiences and adopt patterns of success [9]. Fundamental understanding of synthesis, processing and characterisation of functional nanosystems can render exceptional opportunities for their technological transformation to real applications. The dissemination of knowledge on nanotechnology, nanomaterials and their use is now a necessary step by considering the enormous potential applications that nanotechnologies offer in terms of materials development, techniques and uses. Including both new construction and Cultural Heritage, nanotechnology is providing a significant input to innovation in processes and products. There are numerous research studies and experimentations relating to technological innovation for the preservation and appreciation of the ancient built heritage. In particular, the deterioration of stone and wood with which the ancient structures and monuments are built is a complex physical-chemical process caused by the interaction of several factors: climate of the locations, urban pollution, and the same material properties.

Every direct method to consolidate and, more particularly, to protect and conserve the ancient structures must have the following characteristics: a) to be respectful of the environment; b) to be careful not to damage the wood surfaces or stone material of the ancient structures; c) not to affect the structural characteristics of the material that forms the structure to be preserved; d) to be well absorbed by capillarity; e) not to produce any change of colour of the treated material; f) to have a good penetration and ensure a high degree of consolidation. Scientific experiences of the Author of the present contribution, shown by scientific and technological patents, regarding the implementation projects on nanomaterials, nanostructured inorganic oxides, and more particularly titanium sesquioxide (Ti2O3) and silicon (Si2O3), have demonstrated that it is possible to obtain materials with a high level of biocompatibility that can be used for the consolidation of archaeological wood and stones. One of the most promising inorganic compounds for the consolidation of these materials is lithium silicate.

At the state of the art, two patented inventions in the United States, respectively US n. 4.443 .496 (Obitsu et al., Application No. 400,820) and US n. 4.521.249 (Obitsu et al., Application No. 567,028), claim the use of lithium silicate to impregnate concrete surfaces. The first invention (1984) is titled Agent and method for modifying surface layer of cement structures and the second invention (1985) is titled Silicate containing agent cement surface modified with this agent. The descriptions of said patents highlight a method for the formation of a silicate coating on the surface of concrete. Actually, the inventions claimed by the above-mentioned U.S. patents do not address the problem of consolidation and conservation of stone, of which the $\mathrm{pH}$ is much lower than the matrix of cement.

The patents provide for the use of a plasticizer which is sodium salt of naphthalene-sulfonate condensed with formaldehyde. This polymer has a typical dark brown colour and its application produces an undesirable colour formation of substrate (brown staining). Furthermore, the introduction of said organic material produces a microbial infestation of the stone. Currently, the research aims to constantly create new materials capable of consolidating structures of stone and wood of ancient buildings, without generating any of the aforementioned problems. 


\section{Results and Discussion}

\subsection{Case of Study}

The new patent, invented in 2011 and entitled Innovative sonochemical process that employs ultrasonic cavitation for the synthesis of mono-dispersed amorphous silicon dioxide nanoparticles, and a method for producing high-performance water-soluble lithium silicate compounds, for the application in the consolidation in situ of ancient stone and wood structures (Di Salvo S., Patent Pending PA2011A000012), represents a powerful breakthrough in the synthesis of new materials for the protection of buildings, therefore for the improvement of the built environment. This invention relates to a process for synthesizing amorphous nanoparticles and mono-dispersed of silicon dioxide ( $\mathrm{SiO} 2)$, and a method for preparing a compound water-soluble inorganic compound of lithium silicate by reaction, in water, of lithium hydroxide and/or carbonate with said nanoparticles and mono-dispersed of silicon dioxide. Process and method are in place through the use of ultrasonic energy with a frequency between $10 \mathrm{kHz}$ and 100 $\mathrm{kHz}$. That compound of high-performance lithium silicate is applied in situ for the consolidation of ancient stone and wooden structures. In particular, the innovation of the present invention is that of creating economically and conveniently nanoparticles of silicon dioxide, through a new process of synthesis which employs ultrasonic cavitation, totally unknown in the state of the art of science and technology. Ultrasonic cavitation is the energetic effect which is basically used by ultrasound.

To be more precise, the ultrasonic cavitation is a physical phenomenon consisting in the creation of vacuum "tears" commonly referred to as "bubbles" in a fluid which immediately and violently implode. This compound of silicon dioxide in nanoscale structure can subsequently react with lithium hydroxide and/or carbonate, in water, to form a specific inorganic material of high-performance having new characteristics. The nanoparticles of silicon dioxide have a crucial role in the creation of a new form of water-soluble lithium silicate, to be applied in situ improving the performance of stone and wood materials of ancient structures, essential for a lot of applications in numerous fields of technology. This new material of lithium silicate performs the task perfectly respecting the environment, without changing the physico-chemical and mechanical structures of the treated materials, and all with no lasting effects. This new process belongs to a field of nanoscience and is directed to the creation and utilization of materials, with dimensions at the molecular level, with features not only greatly improved compared to the traditional ones, but even completely innovative.

In fact, the properties and behavior of nanoscale material, obtained according to the teachings of the present invention, provides an opportunity to create inorganic compounds of lithium silicate that operate in a completely different way than those with macro dimensions. In the following example, it is described an economical method for synthesizing nanoparticles of silicon dioxide, through the use of ultrasonic cavitation and use of sodium silicate as a precursor. Further characteristics and advantages of the object of the present invention will become more apparent through the example of a preferred embodiment, but not exclusive, of the invention, described by way of non-limitative example of the nature of the invention.

\subsection{Example of preparation of Silicon Dioxide Nanoparticles}

Under the effect of ultrasonic energy at $40 \mathrm{KHz}$, a solution of concentrated $\mathrm{HCl}(2.0 \%)$ is added dropwise to an aqueous solution of sodium silicate, up to the formation of a colloidal turbid liquid. This precipitate is recovered by filtration and washed, then dried into the oven at $100^{\circ} \mathrm{C}$ for 13 hours and finally calcined in air at $1000^{\circ} \mathrm{C}$ for 40 minutes. The Author of the present invention has found that, for the synthesis of said nanoparticles, very specific parameters must be taken into consideration. These parameters include: ultrasonic cavitation, concentration of sodium silicate and hydrochloric acid concentration.

The nanoparticles, characterized by an average diameter of $\sim 20$ nanometers $(\mathrm{nm})$, are synthesized through the use of the ultrasonic cavitation that determines the optimal conditions for the formation of molecules with a well-defined, regular spherical structure (Figure 1). More particularly, the Author has found that an adequate ultrasonic frequency is indispensable, in order to avoid the agglomeration of the silicate dioxide nanoparticles. To have an optimum cavitation, capable of generating spherical nanoparticles well-defined of silicate dioxide, this ultrasonic frequency must be between $20 \mathrm{KHz}$ and $60 \mathrm{KHz}$. In effect, various experiments have shown that a too high ultrasonic frequency could generate the formation of nanoparticles of silicate dioxide characterized by irregular shapes, while a too low ultrasonic frequency could delay the formation of nanoparticles. Others experiments conducted by the Author have demonstrated that a low concentration of $\mathrm{HCl}$ is an expedient to avoid the agglomeration of the nanoparticles.

The first step for the synthesis of nanoparticles of silicon dioxide is the release of silicic acid $(\mathrm{Si}(\mathrm{OH}) 4)$ through the hydrolysis of sodium silicate with hydrochloric acid according to the following equation: $\mathrm{Na} 2 \mathrm{SiO} 3+\mathrm{H} 2 \mathrm{O}+$ $2 \mathrm{HCl}=\mathrm{Si}(\mathrm{OH}) 4+2 \mathrm{NaCl}$. Subsequently, the silicic acid begins to polymerize with the elimination of the water.

The polymer continues to grow with removal of water forming polymer spheres called primary particles of silicate dioxide, and their magnitude is equal to a few Angstroms (1 Angstrom $=0.1$ nanometers or $1 \times 10-10$ meters $)$. At some point these primary particles begin to stick to each other through the silanol groups $(\mathrm{SiOH})$ to form silica gel ( $\mathrm{SiO} 2)$, produced in the form of hard granules having the appearance of crystals. The silicic acid condenses rapidly to silicon dioxide with removal of water according to the following reaction: $(\mathrm{Si}(\mathrm{OH}) 4=\mathrm{SiO} 2+2 \mathrm{H} 2 \mathrm{O})$. Finally, 
mono-dispersed nanoparticles of amorphous silicon dioxide with diameter of $\sim 20 \mathrm{~nm}$ and a regular spherical structure are synthesized successfully using only a specific ultrasonic cavitation and sodium silicate as precursor. In order to achieve the best conditions for synthesizing these nanoparticles, the reaction temperature should be maintained at $40^{\circ} \mathrm{C}-50^{\circ} \mathrm{C}$ and the ultrasonic frequency of $40 \mathrm{KHz}$.

The present invention also includes the method for the preparation of the new compound of water-soluble lithium silicate, which comprises the following steps: a) to pour distilled water in a stainless steel reactor, thermostated and equipped with a sonicator which generates ultrasound; b) to heat the water to a temperature of $60^{\circ} \mathrm{C}$; c) to trigger ultrasonic energy characterized by a frequency of $40 \mathrm{KHz}$ and pour a predetermined dose of lithium hydroxide; d) to add in small doses nanoparticles of silicon dioxide (synthesized according to the above example); e) after adding the last amount of silicon dioxide, to raise the reactor temperature up to $70^{\circ} \mathrm{C}$; f) when the viscosity of the material contained in the reaction chamber is lowered, to add distilled water and let the solution cool down; g) to take from the reactor the lithium silicate compound $(\mathrm{Li} 2 \mathrm{O} / \mathrm{SiO} 2)$.

This water-soluble compound can be used as a hardener, characterized by the unique ability to penetrate by capillary action in the pores, cracks and lesions of structures of stone and wood. The concentration of lithium silicate in the consolidating product ready for use is less than $20 \%$ by weight, while the ratio $\mathrm{Li} 2 \mathrm{O}: \mathrm{SiO} 2$ is in the range 1.0: 1.0 to $1.0: 20.0$. The Author of this invention has found that the optimum viscosity of the aqueous solution ready to use lithium silicate should be in the range from $1.8 \mathrm{mPas}$ to 2.5 mPas (megapascal).

\section{Claims and Conclusion}

In order to what has been described in detail, the experts in the field can understand that variations in the way of realization of the invention described in the example here shown are possible without thereby alter the technical concepts and/or the essential characteristics of the invention. Therefore, it must be expressly understood that the above example has been proposed only for illustrative and not limitative model of the present invention.

Finally, the invention includes all modifications, additions and substitutions derived from the purpose and spirit of the invention are well declared in the included following claims:

1) Sonochemical process that uses ultrasonic cavitation for the synthesis of silica $(\mathrm{SiO} 2)$ in the form of amorphous mono-dispersed nanoparticles, characterized in that said silicon dioxide is prepared by use of hydrochloric acid $(\mathrm{HCl})$ and sodium silicate as a precursor.

2) Process as claimed in Claim 1, characterized in that the hydrochloric acid concentration should be from $0.1 \%$ to $5.0 \%$.

3) Process as claimed in Claims 1 to 2, characterized in that it uses ultrasonic energy at a frequency between 10 $\mathrm{KHz}$ to $100 \mathrm{KHz}$, and more particularly between $20 \mathrm{KHz}$ and $40 \mathrm{KHz}$.

4) Process as claimed in Claims 1 to 3, characterized in that the size of silica nanoparticles can be controlled by varying the frequency of the ultrasonic energy.

5) Process as claimed in Claims 1 to 4 , characterized in that the mono-dispersed silica nanoparticles have sizes ranging from $1 \mathrm{~nm}$ to $100 \mathrm{~nm}$ and more particularly from $10 \mathrm{~nm}$ to $50 \mathrm{~nm}$.

6) Process as claimed in Claims 1 to 5, characterized in that these silica nanoparticles can react with lithium hydroxide and/or carbonate in aqueous solution to obtain water-soluble lithium silicate with nanoscale structure.

7) Process as claimed in Claim 6, characterized in that said water-soluble lithium silicate can be applied in situ for the consolidation and protection of stone and wooden of ancient structures.

8) Method for preparing a water-soluble inorganic compound of lithium silicate by use of ultrasonic energy having a frequency between $10 \mathrm{kHz}$ and 100 $\mathrm{kHz}$, characterized in that said lithium silicate compound is obtained by reaction, in water, of lithium hydroxide and/or carbonate with silica nanoparticles synthesized according to the above Claims 1 to 5 .

9) Method as claimed in Claim 8, characterized in that for the preparation of said water-soluble lithium silicate can be used ultrasonic energy having a frequency between $20 \mathrm{KHz}$ and $60 \mathrm{KHz}$.

10) Method as claimed in Claim 9, characterized in that said water-soluble lithium silicate can be present in aqueous solution with a maximum solids content of 40 wt. $\%$ and a max viscosity of $20 \mathrm{mPas}$.

11) Method as claimed in Claims 8 to 10 , characterized in that the consolidation of natural stones, historic plasters and wooden structures, is produced in situ using an aqueous solution of lithium silicate, whose maximum concentration is less than $20 \mathrm{wt} \%$ and is characterized by a Li2O:SiO2 ratio of 1.0: $1.0-20.0$.

12) Method as claimed in Claims 8 to 11 , characterized in that the aqueous solution consisting of lithium silicate has maximum concentration less than $10 \mathrm{wt} \%$.

The consolidating power of water-soluble lithium silicate is highly dependent on the size of the amorphous and mono-dispersed nanoparticles of silicon dioxide. The sonochemical technique, which uses ultrasonic cavitation, has the following advantages in the synthesis of nanoparticles of silicon dioxide: versatility and ease of execution, purity, consistency and high performance of the material obtained. In fact, the self-cleaning ability of this new type of silicon dioxide, having a nanoscale structure, applied directly to the stone and wooden surfaces, allows us to preserve their condition unchanged, without any alterations to their appearance of technical features, preventing biological pollutants and corrosion impurities, effectively counteracting the deterioration of the surfaces of 
stone materials [10].

This invention, with numerous studies in the field, demonstrate a commitment and interest in the research for innovative materials and the most reliable systems to ensure the consolidation and restoration of ancient structures, significantly reducing maintenance costs. Several lines of research have developed innovative methods and new production processes that allow nanostructured materials to become an advantageous and indispensable component for the preservation, enhancement and appreciation of the ancient built environment. These new nanomaterials can be successfully tested and verified to help bringing history to life, to protect our built environment, setting the stage for a really accessible and safeguarded city in the future.

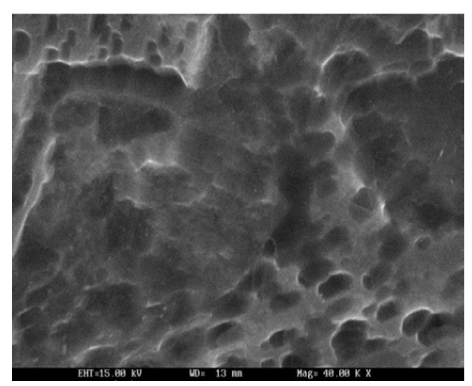

Figure 1. Image SEM (Scanning Electron Microscopy). Morphology of the surface of a porous stone sprayed with a $10 \%$ solution of lithium silicate which contains nanoparticles of silicon dioxide $(\sim 20 \mathrm{~nm})$.

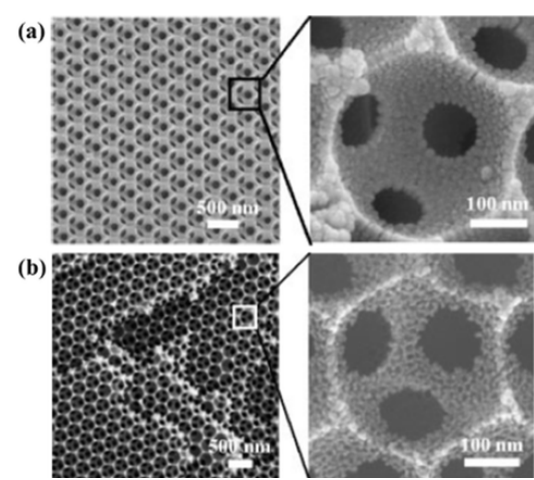

Figure 2. SEM images at different magnifications of a silica template composed of silica nanoparticles.

\section{References}

[1] Brandon, P. and Lombardi, P., Evaluating Sustainable Development in the Built Environment, Wiley-Blackwell, Oxford, United Kingdom, 2011.

[2] Stubbs J. H and Makaš E. G., Architectural conservation in Europe and the Americas, Wiley, Hoboken, 2011.

[3] Pearce J., Albritton S., Grant G., Steed G., \& Zelenika I., A new model for enabling innovation in appropriate technology for sustainable development, Sustainability: Science, Practice, \& Policy 8(2), 2012, pp. 42-53.

[4] Ayong Le Kama, A. D., "Sustainable growth renewable resources, and pollution", Journal of Economic Dynamics and Control 25/12, 2001, pp. 1911-1918.

[5] Macinnes, L., Historic Landscape Characterization, in Bishop and Phillips (eds) Countryside Planning: New approaches to Management and Conservation, Earthscan, London, 2004, pp. 155-169.

[6] Matero, F. G. \& Teutonico, J. M., Managing change: sustainable approaches to the conservation of the built environment. 4th Annual US/ICOMOS International Symposium organized by US/ICOMOS, Program in Historic Preservation of the University of Pennsylvania, and the Getty Conservation Institute Philadelphia, 6-8 April 2001.

[7] Hubbert, M. K, Daniels, F. \& Wigner, E. P., Our Energy Resources. Physics Today 2 (April 1949), 19-22.

[8] Bennet, G., Cultural Landscape. The conservation challenge in a changing Europe. Institute for European Environmental Policy, London, 1996.

[9] Fairclough, G., Protecting Time and Space: undestanding historic landscape for conservation in England, in Ucko, P. J. \& Layton, R. (eds), The Archaeology and Anthropology of Landscape: Shaping your landscape, One World Archaeology 30, Routledge, London, pp. 119-134.

[10] Di Salvo, S., Methodological approaches for the enhancement of cultural heritage, Aracne, Rome, 2012. 